\title{
ORIGINAL
}

\section{A novel homozygous mutation of the nicotinamide nucleotide transhydrogenase gene in a Japanese patient with familial glucocorticoid deficiency}

\author{
Rie Yamaguchi ${ }^{1)}$, Fumiko Kato ${ }^{1)}$, Tomonobu Hasegawa ${ }^{2)}$, Noriyuki Katsumata ${ }^{3)}$, Maki Fukami ${ }^{3)}$, \\ Toshiharu Matsui ${ }^{4)}$, Keisuke Nagasaki ${ }^{5)}$ and Tsutomu Ogata ${ }^{1)}$ \\ 1) Department of Pediatrics, Hamamatsu University School of Medicine, Hamamatsu 431-3192, Japan \\ 2) Department of Pediatrics, Keio University School of Medicine, Tokyo 160-8582, Japan \\ 3) Department of Molecular Endocrinology, National Research Institute for Child Health and Development, Tokyo 157-8535, \\ Japan \\ 4) Department of Pediatrics, Nagaoka Chuo General Hospital, Nagaoka 940-8653, Japan \\ ${ }^{5)}$ Division of Pediatrics, Niigata University Graduate School of Medicine and Dental Sciences, Niigata 951-8122, Japan
}

\begin{abstract}
Familial glucocorticoid deficiency (FGD) is a rare autosomal recessive disorder characterized by primary hypocortisolism and normal mineralocorticoid production. Recently, $N N T$ encoding the nicotinamide nucleotide transhydrogenase has been identified as a causative gene for FGD. Thus, we examined $N N T$ in six Japanese FGD patients with no recognizable mutation in the previously known four responsible genes for FGD (MC2R, MRAP, STAR, and MCM4), and identified a novel homozygous substitution (c.644T $>$ C; p.Phe215Ser) in a single 17.5-year-old boy. His parents were heterozygous for this mutation. This substitution was absent from 120 Japanese control subjects and was not registered in public databases including JSNP Database. The phenylalanine residue at the 215 th codon was evolutionally conserved, and the p.Phe215Ser was assessed to be a pathologic mutation by in silico protein function analyses. The results, in conjunction with the previous data, imply that NNT mutations account for 5-10\% of FGD patients, and that underlying factor(s) still remains to be clarified in a substantial fraction of FGD patients.
\end{abstract}

Key words: Familial glucocorticoid deficiency, NNT, In silico functional analysis

FAMILIAL GLUCOCORTICOID DEFICIENCY (FGD) is a rare autosomal recessive disorder resulting from defective glucocorticoid biosynthesis in response to ACTH stimulation [1]. Mineralocorticoid production remains normal. The term FGD is conventionally applied to sporadic as well as familial cases with this condition. Salient features of FGD include hyperpigmentation, hypoglycemia, and failure to thrive [1]. Previous studies in FGD have identified multiple mutations of $M C 2 R$ encoding the melanocortin-2 (ACTH) receptor in $c a .25 \%$ of patients, plural mutations of $M R A P$ encoding the MC2R accessory protein in $c a$. $20 \%$ of patients, certain mutations of STAR encoding the steroidogenic acute regulatory protein in $c a .5 \%$

Submitted Jan. 15, 2013; Accepted Feb. 21, 2013 as EJ13-0024 Released online in J-STAGE as advance publication Mar. 9, 2013

Correspondence to: Tsutomu Ogata, Department of Pediatrics, Hamamatsu University School of Medicine, 1-20-1 Handayama, Higashi-ku, Hamamatsu 431-3192, Japan.

E-mail: tomogata@hama-med.ac.jp

(C) The Japan Endocrine Society of patients, and a single mutation of MCM4 encoding mini chromosome maintenance-deficient 4 homologue in the Irish Traveler population [reviewed in ref. 1]. However, no mutation has been found in $c a .50 \%$ of patients, suggesting the presence of hitherto unidentified responsible genes in FGD.

Recently, Meimaridou et al. have demonstrated that FGD is caused by various nonsense, frameshift, and missense mutations in NNT for nicotinamide nucleotide transhydrogenase [2]. NNT consists of 22 exons and encodes a 1,086 amino acid protein that harbors the $\mathrm{NAD}(\mathrm{H})$-binding domain, the transmembrane domain, and the NADP(H)-binding domain, and functions as a proton-translocating enzyme of the inner mitochondrial membrane. Meimaridou et al. first localized a candidate region to chromosome 5p13-q12 encompassing NNT by genotyping for consanguineous families and, subsequently, identified a total of 21 different $N N T$ mutations in 15 kindreds with FGD by analyzing $c a .100$ FGD patients without a demonstrable muta- 
tion in $M C 2 R, M R A P$, and STAR. Furthermore, Nnt knockout mice exhibited excessive apoptosis in zona fasciculata cells and compromised basal and stimulated corticosterone levels, and $N N T$ knockdown resulted in impaired redox potential and increased reactive oxygen species (ROS) in an H295R human adrenocortical tumor cell line [2]. Thus, while direct functional studies have not been performed for NNT mutations, these findings indicate that $N N T$ mutations lead to FGD probably due to defective ROS detoxification.

In this regard, we have previously examined 10 Japanese patients with FGD, and identified an $M C 2 R$ mutation in a single patient and STAR mutations in three patients (no mutation has been found in MRAP and MCM4) [ref. 3 and our unpublished observation]. Thus, we analyzed $N N T$ in the remaining six patients with no recognizable mutation in the four genes, and identified a novel homozygous $N N T$ mutation in a single patient.

\section{Case Report}

This Japanese male patient was born to apparently non-consanguineous parents at 39 weeks of gestation with a birth weight of $3.1 \mathrm{~kg}(-0.4 \mathrm{SD})$, after an uncomplicated pregnancy and delivery. The parents were clinically normal, as were the elder brother and sister.

At 19 months of age, he was referred to us because of generalized skin pigmentation. His length was $82.0 \mathrm{~cm}(+0.2 \mathrm{SD})$, and his weight $10.6 \mathrm{~kg}( \pm 0 \mathrm{SD})$. Physical examination showed a somewhat feeble boy with no dysmorphic features. External genitalia were well masculinized with bilateral intrascrotal testes. His blood pressure was $95 / 60 \mathrm{mmHg}$. Blood glucose was $86 \mathrm{mg} / \mathrm{dL}, \mathrm{Na} 134 \mathrm{mEq} / \mathrm{L}, \mathrm{K} 4.1 \mathrm{mEq} / \mathrm{L}$, and Cl 102 $\mathrm{mEq} / \mathrm{L}$. Endocrine studies revealed primary hypocortisolism without increased $17-\mathrm{OH}$ progesterone (Table 1). Serum aldosterone was low-normal, while plasma renin activity was elevated. Abdominal computed tomography failed to delineate adrenal glands. On the basis of the above findings (i.e., primary hypocortisolism, no accumulation of $17-\mathrm{OH}$ progesterone, no definitive mineralocorticoid deficiency, and normal male sex development), he was diagnosed as having FGD, and was placed on oral hydrocortisone replacement therapy $\left(20-30 \mathrm{mg} / \mathrm{m}^{2} /\right.$ day $)$.

Subsequent clinical course was uneventful with normal blood glucose and electrolyte values, although he sometimes felt tiredness after school. On the last examination at 17.5 years of age, he measured $163.2 \mathrm{~cm}$ $(-1.3 \mathrm{SD})$, weighed $52.0 \mathrm{~kg}(-1.0 \mathrm{SD})$, and manifested age-appropriate secondary sexual characteristics (pubic hair, Tanner stage 4). Blood endocrine data were grossly normal, although dehydroepiandrosterone was slightly low and rennin activity was slightly high (Table 1). Blood HbAlc was 4.9\% (reference range: 4.3 - 5.8),

Table 1 Blood hormone data

\begin{tabular}{|c|c|c|c|c|c|c|}
\hline & \multirow[b]{2}{*}{ Unit } & \multicolumn{3}{|c|}{19 months (morning) ${ }^{\mathrm{a}}$} & \multicolumn{2}{|c|}{17.5 years (morning) ${ }^{b}$} \\
\hline & & Baseline & (Reference) & Stimulated & Baseline & (Reference) \\
\hline ACTH & $\mathrm{pg} / \mathrm{mL}$ & 3513 & $(18.7 \pm 6.8)$ & $\ldots$ & 5.0 & $(4.4-48.0)$ \\
\hline Cortisol & $\mu \mathrm{g} / \mathrm{mL}$ & 1.6 & $(12.2 \pm 5.8)$ & $1.5^{\mathrm{c}}$ & $\ldots$ & $(2.7-15.5)$ \\
\hline Pregnenolone & $\mathrm{ng} / \mathrm{mL}$ & $<0.10$ & $(<0.1-0.55)$ & $<0.10^{\mathrm{c}}$ & $\ldots$ & \\
\hline 17-OH progesterone & $\mathrm{ng} / \mathrm{mL}$ & 0.6 & $(0.90 \pm 1.01)$ & $0.7^{\mathrm{c}}$ & $\ldots$ & \\
\hline Dehydroepiandrosterone & $\mathrm{ng} / \mathrm{mL}$ & 0.27 & $(0.39 \pm 0.09)$ & $0.28^{\mathrm{c}}$ & 0.71 & $(2.3-9.2)$ \\
\hline Deoxycorticosterone & $\mathrm{ng} / \mathrm{dL}$ & 0.24 & $(<0.03-0.33)$ & $0.16^{\mathrm{c}}$ & $\ldots$ & \\
\hline Aldosterone & $\mathrm{ng} / \mathrm{dL}$ & 3.9 & $(14.2 \pm 7.6)$ & $4.7^{\mathrm{c}}$ & 7.6 & $(5.7-15.0)$ \\
\hline Renin activity & $\mathrm{ng} / \mathrm{mL} / \mathrm{hr}$ & 19.8 & $(2.11 \pm 1.14)$ & $\ldots$ & 5.2 & $(1.9-3.7)$ \\
\hline $\mathrm{LH}$ & $\mathrm{mIU} / \mathrm{mL}$ & $<0.5$ & $(<0.5-2.0)$ & $1.8^{\mathrm{d}}$ & 2.9 & $(1.8-6.5)$ \\
\hline FSH & $\mathrm{mIU} / \mathrm{mL}$ & 1.0 & $(0.66-2.46)$ & $8.7^{\mathrm{d}}$ & 6.9 & $(3.6-10.5)$ \\
\hline Testosterone & $\mathrm{ng} / \mathrm{dL}$ & 32.5 & $(5-20)$ & $\ldots$ & 326 & $(300-1,017)$ \\
\hline
\end{tabular}

Reference values represent age-matched baseline serum hormone levels.

${ }^{\text {a }}$ Before treatment.

${ }^{\mathrm{b}}$ Under oral hydrocortisone replacement therapy.

${ }^{\mathrm{c}}$ ACTH $250 \mu \mathrm{g} / \mathrm{m}^{2}$ bolus i.v.; blood sampling at 30 and $60 \mathrm{~min}$ (higher values are shown).

${ }^{\mathrm{d}} \mathrm{GnRH} 100 \mu \mathrm{g} / \mathrm{m}^{2}$ bolus i.v.; blood sampling at 30, 60, 90, and $120 \mathrm{~min}$ (peak values are shown). 


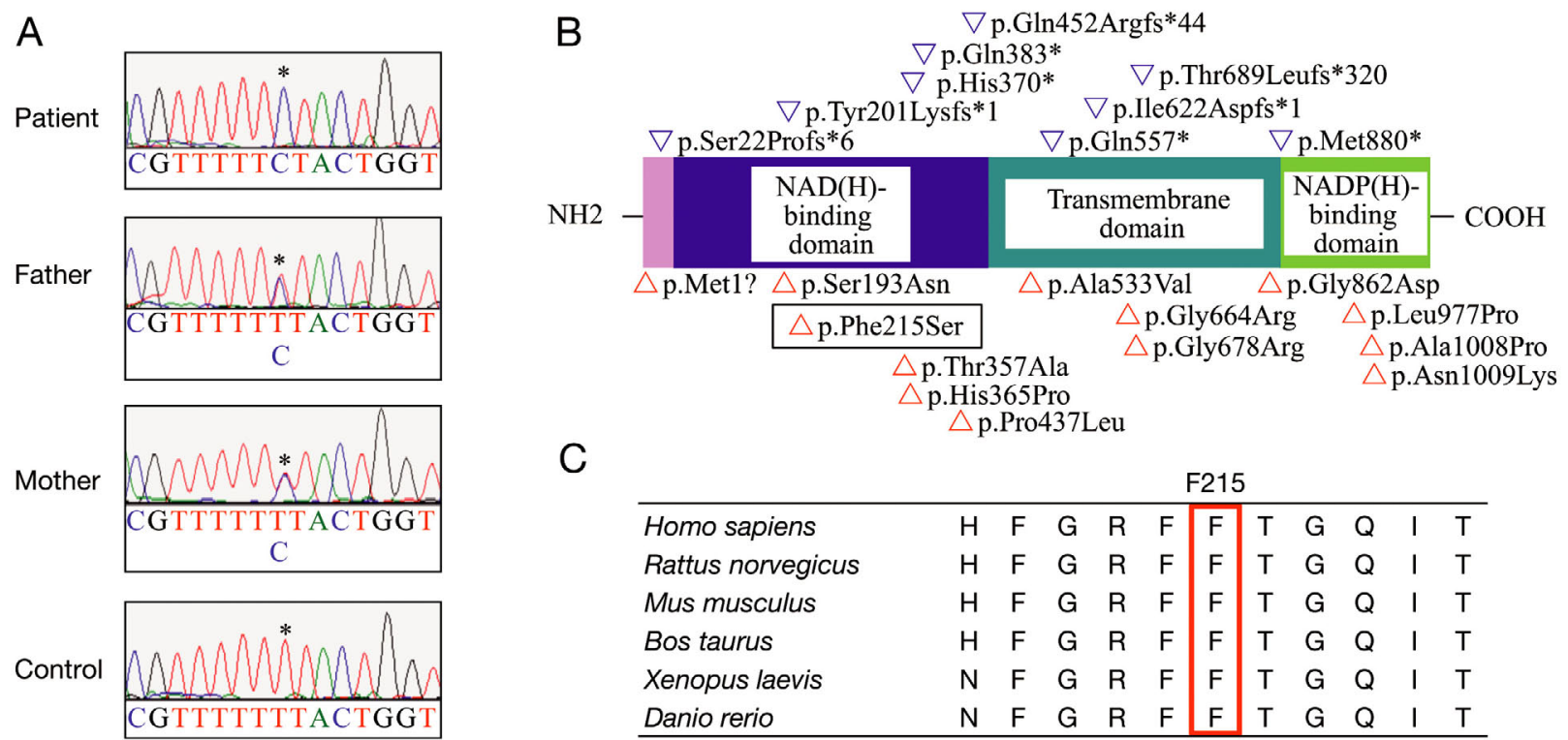

Fig. 1 A novel NNT mutation identified in this patient

A. Direct sequencing analysis showing a homozygous mutation (c.644T $>$ C; p.Phe215Ser) in this patient (indicated with asterisks). The parents are heterozygous for this mutation.

B. The distribution of the NNT mutations reported in Meimaridou et al. [2] and this study. Shown on the upper part and the lower of the diagram for the NNT protein structure are the positions of nonsense and frameshift mutations (denoted with blue downward triangles) and those of missense mutations (indicated with red upward triangles). The p.Phe215Ser identified in this study (surrounded with a rectangle) resides on the NAD $(\mathrm{H})$-binding domain.

C. Evolutional conservation of the phenylalanine residue at the 215 th codon.

lactic acid $10.8 \mathrm{mg} / \mathrm{dL}(4-17)$, and total ketone bodies $38 \mu \mathrm{mol} / \mathrm{L}(<130)$. Mutation analysis failed to identify a pathologic mutation in $M C 2 R, M R A P, S T A R$, and MCM4 for FGD as well as in NROBI (DAXI) and NR5A1 (SF1) for adrenal hypoplasia congenita.

\section{Mutation analysis of NNT}

This study was approved by the Institutional Review Board Committee at Hamamatsu University School of Medicine. After obtaining informed consent, direct sequencing was performed for all the 21 coding exons and their splice sites of $N N T$, using leukocyte genomic DNA of this patient. The primer sequences are available on request.

A novel homozygous missense substitution (c.644T $>$ C, p.Phe215Ser) was identified at exon 5 encoding the $\mathrm{NAD}(\mathrm{H})$-binding domain of this paient (Fig. 1A and B). The parents were subsequently found to be heterozygous for this substitution. This substitution was absent from 120 Japanese control subjects and was not registered in public databases including Japanese SNP Database (http://snp.ims.u-tokyo. ac.jp/). The phenylalanine residue at the 215 th codon was evolutionally conserved (http://www.ncbi.nlm. nih.gov/blast/Blast.cgi) (Fig. 1C), and the p.Phe215Ser was predicted as "damaging" with a score of 0.00 by Sorting Intolerant From Tolerant (SIFT, http://sift. bii.a-star.edu.sg/) [4] and as "probably damaging" with a score of 1.00 (sensitivity 0.00 and specificity 1.00) by Polymorphism Phenotyping-2 (PolyPhen-2, http:// genetics.bwh.harvard.edu/pph2/) [5].

\section{Discussion}

We identified a novel missense substitution of $N N T$ in a patient with FGD. In this regard, lack of this substitution in the control subjects and in the public databases, evolutional conservation of the phenylalanine residue at the 215th codon, and in silico function data for the p.Phe215Ser by SIFT and PolyPhen-2 argue for this substitution being a pathologic mutation. In this context, while the p.Phe215Ser could have either a gain-of-function or a loss-of-function effect, previous studies have identified various NNT nonsense and frameshift mutations, which should lose the normal protein function, as well as various NNT missense 
mutations in patients with FGD [2]. It is predicted, therefore, that the p.Phe215Ser detected in this patients with FGD also has a loss-of-function effect. In addition, while functional studies have not been performed, the position of the p.Phe215Ser on the NAD $(H)$ binding domain would imply that this mutation affects the $\mathrm{NAD}(\mathrm{H})$ binding. To our knowledge, this is the second report describing an NNT mutation in FGD.

This mutation was identified in one of six FGD patients with no recognizable mutation in $M C 2 R$, $M R A P, S T A R$, and MCM4. The frequency is grossly similar to that reported by Meimaridou et al. who identified NNT mutations in 15 of $c a .100$ FGD patients without a demonstrable mutation in $M C 2 R, M R A P$, and $S T A R$ [2]. Since the mutations in $M C 2 R, M R A P$, and $S T A R$ have been found in $c a .50 \%$ of patients with FGD, and the mutation of MCM4 is extremely rare [1], it is inferred that NNT mutations account for $5-10 \%$ of FGD patients, and that underlying factor(s) still remain to be clarified in a substantial fraction of FGD patients.

Clinical phenotype of this patient is consistent with FGD, as has been indicated for the previously reported patients with $N N T$ mutations [2]. Indeed, while blood $\mathrm{Na}$ was slightly low and rennin activity was slightly high at 19 months of age, this could be explained by lack of cortisol with weak but some degree of mineralocorticoid activity [6]. Thus, although it remains to be clarified why $N N T$ mutations affecting mitochondrial function result in FGD alone, adrenocortical cells at the zona fasciculata producing a large amount of cortisol may specifically be vulnerable to oxidative stress caused by impaired redox potential and increased ROS. However, since this patient had slightly high blood rennin activity and slightly low dehydroepiandrosterone value at 17.5 years of age, subclinical dysfunction may also lie in adrenocortical cells at the zona glomerulosa and the zona reticularis. Similarly, the low-normal blood testosterone value at 17.5 years of age might be related to the NNT mutation, because ROS-mediated mitochondrial dysfunction is known to inhibit STAR protein function and steroidogenesis in Leydig cells [7]. Furthermore, while blood glucose, lactic acid, and whole ketone bodies were normal in this patient, Nnt deficient $\mathrm{C} 57 \mathrm{BL} / 6 \mathrm{~J}$ mice show reduced insulin secretion and high-fat diet-induced diabetes mellitus, in addition to adrenal dysfunction $[8,9]$. Thus, abnormal glucose metabolism might be present in exceptional patients with $N N T$ mutations. In this regard, it is notable that NNT gene was studied in FGD phenotype-positive patients with apparently heterogeneous genetic backgrounds, whereas phenotypic consequence was examined in $N n t$ knockout experimental mice with a considerably homogeneous genetic background. Such differences in the employed research method and in the genetic background would primarily explain phenotypic difference between $N N T$ mutation positive patients and Nnt knockout mice.

In summary, we identified a Japanese patient with FGD due to a novel $N N T$ mutation. Further studies will permit to elucidate the role of NNT mutations in the development of FGD and to define phenotypic spectrum in patients with $N N T$ mutations.

\section{Disclosure Summary}

All authors have nothing to disclose.

\section{Acknowledgements}

This work was supported in part by Grant for Research on Intractable Diseases from the Ministry of Health, Labor and Welfare (H24-048), by Grant from National Center for Child Health and Development (23A-1), and by Grant-in-Aid for Scientific Research on Innovative Areas (22132004) from the Ministry of Education, Culture, Sports, Science and Technology (MEXT).

\section{References}

1. Meimaridou E, Hughes CR, Kowalczyk J, Guasti L, Chapple JP, et al. (2013) Familial glucocorticoid deficiency: New genes and mechanisms. Mol Cell Endocrinol, 2012 Dec 29 [Epub ahead of print].

2. Meimaridou E, Kowalczyk J, Guasti L, Hughes CR, Wagner F, et al. (2012) Mutations in NNT encoding nicotinamide nucleotide transhydrogenase cause familial glucocorticoid deficiency. Nat Genet 44: 740-742.
3. Ishii T, Ogata T, Sasaki G, Sato S, Kinoshita EI, et al. (2000) Novel mutations of the ACTH receptor gene in a female adult patient with adrenal unresponsiveness to ACTH. Clin Endocrinol (Oxf) 53: 389-392.

4. Kumar P, Henikoff S, Ng PC (2009) Predicting the effects of coding non-synonymous variants on protein function using the SIFT algorithm. Nat Protoc 4: 10731081 . 
5. Adzhubei IA, Schmidt S, Peshkin L, Ramensky VE, Gerasimova A, et al. (2010) A method and server for predicting damaging missense mutations. Nat Methods 7: 248-249.

6. Stewart PM, Krone NP (2011) The adrenal cortex. In: Melmed S, Polonsky KS, Larsen PR, Kronenberg HM (eds). Williams Textbook of Endocrinology $\left(12^{\text {th }}\right)$. Elsevier Saunders, Philadelphia: 479-544.

7. Diemer T, Allen JA, Hales KH, Hales DB (2003) Reactive oxygen disrupts mitochondria in MA-10 tumor Leydig cells and inhibits steroidogenic acute regulatory
(StAR) protein and steroidogenesis. Endocrinology 144: 2882-2891.

8. Toye AA, Lippiat JD, Proks P, Shimomura K, Bentley $\mathrm{L}$, et al. (2005) A genetic and physiological study of impaired glucose homeostasis control in C57BL/6J mice. Diabetologia 48: 675-686.

9. Fueger PT, Bracy DP, Malabanan CM, Pencek RR, Granner DK, et al. (2004) Hexokinase II overexpression improves exercise-stimulated but not insulin-stimulated muscle glucose uptake in high-fat-fed C57BL/6J mice. Diabetes 53: 306-314. 